\title{
Correlation Between Tumor Metabolism and Semiquantitative Perfusion MRI Metrics in Non-small Cell Lung Cancer
}

\author{
Sang Ho Lee, $\mathrm{PhD}^{1}$, Andreas Rimner, $\mathrm{MD}^{2}$, Emily Gelb, BS ${ }^{2}$, Joseph O. Deasy, PhD ${ }^{1}$, \\ Margie A. Hunt, $\mathbf{M S}^{1}$, John L. Humm, PhD $^{1}$, and Neelam Tyagi, PhD $^{1}$ \\ ${ }^{1}$ Department of Medical Physics, Memorial Sloan-Kettering Cancer Center, New York, NY 10069 \\ ${ }^{2}$ Department of Radiation Oncology, Memorial Sloan-Kettering Cancer Center, New York, NY \\ 10069
}

\section{Abstract}

Purpose-To correlate semiquantitative parameters derived from dynamic contrast-enhanced MRI (DCE-MRI) and ${ }^{18}$ F-FDG-PET for non-small cell lung cancer (NSCLC).

Methods-Twenty-four NSCLC patients who underwent pretreatment ${ }^{18}$ F-FDG-PET and DCEMRI were analyzed. The maximum standardized uptake value (SUVmax) was measured from ${ }^{18} \mathrm{~F}$ FDG-PET. DCE-MRI was obtained on 3T MRI scanner using four-dimensional T1-weighted highresolution imaging with volume excitation sequence. DCE-MRI parameters consisting of mean, median, standard deviation (SD), and median absolute deviation (MAD) of peak enhancement, time-to-peak (TTP), time-to-half-peak (TTHP), wash-in slope (WIS), wash-out slope (WOS), initial gradient, wash-out gradient, signal enhancement ratio, and initial area under the relative signal enhancement curve taken up to 30, 60, 90, 120, 150, and $180 \mathrm{~s}$, TTP, and TTHP (IAUCtthp) were calculated for each lesion. Univariate analysis (UVA) was performed using Spearman correlation. A linear regression model to predict SUVmax from DCE-MRI parameters was developed by multivariate analysis (MVA) using least absolute shrinkage selection operator in combination with leave-one-out cross-validation (LOOCV).

Results-In UVA, mean(WOS) $(\rho=-0.456, p=0.025)$, mean(IAUCtthp) $(\rho=-0.439, p=$ $0.032)$, median(IAUCtthp) $(\rho=-0.543, p=0.006)$, and MAD(IAUCtthp) $(\rho=-0.557, p=0.005)$ were statistically significant; all these parameters were negatively correlated with SUVmax. In MVA, a linear combination of SD(WIS), SD(TTP), MAD(TTHP), and MAD(IAUCtthp) was statistically significant for predicting SUVmax (LOOCV-based adjusted $\mathrm{R}^{2}=0.298, \mathrm{p}=0.0006$ ). A decrease in $\mathrm{SD}$ (WIS), MAD(TTHP), and MAD(IAUCtthp) and an increase in $\mathrm{SD}$ (TTP) were associated with a significant increase in SUVmax.

Corresponding Author: Neelam Tyagi, PhD, Department of Medical Physics, Memorial Sloan-Kettering Cancer Center, New York, New York, tyagin@mskcc.org, Ph: (212)-639-2957.

Conflict of Interest: None

Publisher's Disclaimer: This is a PDF file of an unedited manuscript that has been accepted for publication. As a service to our customers we are providing this early version of the manuscript. The manuscript will undergo copyediting, typesetting, and review of the resulting proof before it is published in its final citable form. Please note that during the production process errors may be discovered which could affect the content, and all legal disclaimers that apply to the journal pertain. 
Conclusion-Association was found between SUVmax, the SD, and MAD of DCE-MRI metrics derived during contrast uptake in NSCLC, reflecting that intratumoral heterogeneity in wash-in contrast kinetics is associated with tumor metabolism. Although MAD(IAUCtthp) was a significant feature in both UVA and MVA, the LASSO-based multivariate regression model yielded better predictability of SUVmax than a univariate regression model using MAD(IAUCtthp). This study will facilitate understanding of the complex relationship between tumor vascularization and metabolism, and eventually help in guiding targeted therapy.

\section{Keywords}

DCE-MRI; FDG-PET; non-small cell lung cancer; semiquantitative parameter analysis; vascularmetabolic relationship

\section{Introduction}

Lung cancer is the leading cause of cancer-related mortality in the world in both men and women [1]. Non-small cell lung cancer (NSCLC) is the most common type, accounting for $75-80 \%$ of lung cancer cases [2]. Thus, accurate diagnosis of these lesions is very crucial. ${ }^{18} \mathrm{~F}$-FDG-PET is widely used in the disease management of patients with lung cancer. ${ }^{18} \mathrm{~F}$ FDG-PET exploits the fundamental differences in glucose metabolism between tumor and normal tissue and has been considered useful in distinguishing between benign and neoplastic processes. The most widely used metric of FDG uptake within tumor tissue is the standardized uptake value (SUV), which is the differential uptake ratio between the tumor tissue of interest relative to the uptake were the FDG to be uniformly distributed throughout the body. ${ }^{18}$ F-FDG-PET and the SUV unit have been proven to be of great value in the initial diagnosis [3], detection of recurrent disease [4], evaluation of response to therapy [5], and radiation treatment planning [6].

Tumor growth usually depends on angiogenesis, which plays a critical role in the progression [7], metastasis, and therapy response [8] for the majority of NSCLC. Dynamic contrast-enhanced MRI (DCE-MRI) is a noninvasive method for characterizing the vascularity of tumors [9]. DCE-MRI can be used to obtain a series of sequential T1weighted MR images before, during, and after a bolus injection of a paramagnetic gadolinium-based contrast agent (CA) with rapid acquisition methods. The acquisition process reflects the passage of CA through a particular tissue of interest and offers the possibility to estimate a number of various hemodynamic parameters directly related to angiogenesis [10]. DCE-MRI studies employing simple semiquantitative metrics have become essential clinical tools in oncologic applications because of the simplicity of the approach [11]. A large number of semiquantitative parameters can be extracted on a voxelby-voxel basis along the temporal dimension of DCE-MRI and used to characterize spatiotemporal heterogeneity within the tumor.

Because metabolism sufficient for tumor growth requires an adequate delivery of glucose and oxygen, a well-developed blood supply to the tumor is essential. However, tumor glucose metabolism is also stimulated by hypoxia in which tumor blood flow is insufficient. As a result, the relationship between vascular physiology and glucose metabolism is not 
known for tumors, though it is tightly coupled in normal tissues [12]. Therefore, one of the rationales for this study was to investigate imaging metrics in an attempt to couple vascularmetabolic events and tumor adaptation to the microenvironment [13].

The goal of this study was to investigate the relationship between tumor vascularity, measured by various semiquantitative DCE-MRI parameters, and glucose metabolism, measured by ${ }^{18} \mathrm{~F}-\mathrm{FDG}$-PET in NSCLC.

\section{Materials and Methods}

\section{A. Patient Characteristics}

Twenty-four patients with NSCLC (stage I or II, $\mathrm{n}=8$; stage III or IV, $\mathrm{n}=16$ ) undergoing definitive chemoradiation were included in this IRB-approved study. All patients underwent pretreatment ${ }^{18} \mathrm{~F}$-FDG-PET/CT and DCE-MRI scans. There were 14 men and 10 women (mean age, 65.25 years [range, 40 to 89]; mean weight $77.33 \mathrm{~kg}$ [range, 47 to 113]). Histopathologic subtypes of the NSCLC patients were biopsy-confirmed and included adenocarcinoma ( $\mathrm{AC}, \mathrm{n}=12$ ), squamous cell carcinoma ( $\mathrm{SCC}, \mathrm{n}=10)$, adenosquamous carcinoma (ASCC, $\mathrm{n}=1$ ) and NSCLC not otherwise specified (NOS, $\mathrm{n}=1$ ). Patient characteristics are summarized in Table 1.

\section{B. ${ }^{18} \mathrm{~F}-\mathrm{FDG}-\mathrm{PET} / \mathrm{CT}$}

For PET/CT, $12 \mathrm{mCi}$ of ${ }^{18} \mathrm{~F}-\mathrm{FDG}$ was injected into the patient in the fasted state (or after 6 h) nominally $1 \mathrm{~h}$ before the scan. ${ }^{18} \mathrm{~F}$-FDG-PET scans were acquired as part of a standard clinical whole-body protocol on a GE Discovery ST PET/CT in 3D mode. The protocol consists of acquiring a low dose whole body CT $(80 \mathrm{~mA}$ at $120 \mathrm{kVp})$ followed by the ${ }^{18} \mathrm{~F}-$ FDG-PET scan using a dwell time of $3 \mathrm{~m}$ per bed and an 11-slice overlap (approximately 16 $\mathrm{cm}$ per bed) from mid-skull to upper thighs. PET images were reconstructed using $2 \times 20$ iterations and subsets, with axial 3-point smoothing (Heavy, [l 121$] / 4)$, and a transaxial Gaussian post-filter ( $6.0 \mathrm{~mm}$ full width at half maximum). Tracer uptake was represented by SUV normalized by body weight. Tumor regions of interest (ROIs) on PET were segmented in MIM $^{\mathrm{TM}}$ VISTA using a gradient-based autosegmentation method, and SUVmax was extracted from each tumor ROI. The median SUVmax for the population was 17.90 (range, 2.710 to 23.54$)$.

\section{DCE-MRI Protocol}

MR image acquisition was performed on a 3T Philips Ingenia scanner. DCE-MRI was obtained using a coronal four-dimensional (4D) T1-weighted high-resolution imaging method with volume excitation (4D THRIVE) sequence during free breathing in order to achieve high temporal resolution. The 4D THRIVE is a modification of the standard threedimensional (3D) T1-weighted gradient echo sequence, which is currently used for DCEMRI acquisition. The 4D THRIVE sequence, along with parallel imaging, makes use of Contrast Enhanced Timing Robust Acquisition (CENTRA) and $k$-space order technique to achieve higher spatiotemporal resolution [14]. For DCE-MRI of the NSCLC patients, a total of 130 dynamic sequences were obtained with 30 coronal slices with $2.5 \mathrm{~mm}$ slice thickness, $\mathrm{TR} / \mathrm{TE}$ of $4.2 / 1.9 \mathrm{~ms}$, FA of $15^{\circ}$, in-plane acquisition resolution of $2.5 \mathrm{~mm}$, and temporal 
resolution of $2 \mathrm{~s}$. The bolus injected was $0.2 \mathrm{~mL} / \mathrm{kg}$ of gadolinium-

diethylenetriaminepentaacetic acid (Gd-DTPA, Magnevist) followed by saline flush.

\section{Image Processing and Analysis}

A groupwise image registration method was implemented for compensating potential misalignment in DCE-MRI based on principal component analysis, which makes use of the fact that intensity changes in DCE-MRI can be described by a low-dimensional signal model $[15,16]$. In such an approach, all DCE-MRI time-points were simultaneously registered to a mean space. ROIs covering the entire tumor volume were drawn by an experienced radiation oncologist [A.R.]. Linearity between MR signal and CA concentration was assumed. A relative signal enhancement (RSE) curve was extracted from an ROI placed manually on the pulmonary artery for each patient. The first-pass bolus arrival time (BAT) in the arterial input function (AIF) was automatically identified by fitting the AIF model to the RSE curve in the pulmonary artery [17]. Then, delay correction of the contrast BAT was applied for each voxel within the tumor to shift its RSE curve forward in time by the BAT of the AIF. This allows for accurately estimating the RSE curve shapes from the onset time of CA enhancement, though transit time delay from the AIF to the tumor was not estimated for the sake of simplicity. The intratumoral RSE curves were smoothed temporally using a cubic smoothing spline for noise reduction. Sixteen semiquantitative parameters were calculated from each voxel-wise intratumoral RSE curve: peak enhancement (PE), wash-in-slope (WIS), wash-out-slope (WOS), time-to-peak (TTP), time-to-half-peak (TTHP), initial gradient (IG), wash-out gradient (WG), signal enhancement ratio (SER), and initial area under the curve (IAUC) taken up to 30 (IAUC30), 60 (IAUC60), 90 (IAUC90), 120 (IAUC120), 150 (IAUC150), and $180 \mathrm{~s}$ (IAUC180), TTP (IAUCttp), and TTHP (IAUCtthp) [18-22]. The definitions and physical units of these semiquantitative DCE-MRI metrics are summarized in Table 2. In addition, a more detailed description of these semiquantitative parameters is provided as a supplementary document (supplementary document 1 ). To characterize the spatiotemporal heterogeneity of intratumoral RSE pattern, four spatial features consisting of mean, median, standard deviation (SD), and median absolute deviation (MAD) for the 16 semiquantitative parameter maps were extracted for each patient thus generating a total of $64(=4 \times 16)$ spatiotemporal DCE-MRI features.

\section{E. Statistical Analysis}

Univariate analysis (UVA) was performed using Spearman correlation to investigate correlations between the DCE-MRI features and ${ }^{18}$ F-FDG-PET SUVmax. A $p$ value less than 0.05 was considered statistically significant. Because no features were statistically significant after applying Bonferroni's correction, the UVA was conducted without multiple testing corrections to evaluate as many potential features as possible with subsequent multivariate analysis (MVA). DCE-MRI features with $p<0.1$ on the UVA were identified and returned into the MVA based on the least absolute shrinkage and selection operator (LASSO) [23]. This procedure was combined with leave-one-out cross-validation (LOOCV) to avoid overfitting in the selection of initial features. The DCE-MRI features, chosen at least once in the LOOCV procedure, were then used in the LASSO regression for building a final model, which included an additional feature selection stage combined with the LOOCV. Finally, a regression model forming a linear combination of a subset of relevant 
DCE-MRI features was derived and used for the prediction of SUVmax. The accuracy of the LASSO regression model was evaluated by in-sample and out-of-sample (LOOCV) adjusted $\mathrm{R}^{2}$ to achieve an unbiased estimate of model performance. The SUVmax and LASSOselected parameters were compared between different histopathologies and between different tumor stage groups by using the Wilcoxon rank sum test. The best univariate linear regression model was also investigated with the 64 spatiotemporal DCE-MRI features, and its accuracy was compared with that of the LASSO regression model by the in-sample and out-of-sample adjusted $\mathrm{R}^{2}$. All analyses were performed using the statistical software $\mathrm{R}$ 3.3.3.

\section{Results}

\section{A. Univariate Correlation between DCE-MRI Features and SUVmax}

Univariate Spearman rank correlation coefficients between the DCE-MRI features and ${ }^{18} \mathrm{~F}$ FDG-PET SUVmax are listed in Table 3. The SUVmax was significantly negatively correlated with four DCE-MRI features: mean(WOS) $(\rho=-0.456, p=0.025)$, mean(IAUCtthp) $(\rho=-0.439, p=0.032)$, median(IAUCtthp) $(\rho=-0.543, p=0.006)$, and MAD(IAUCtthp) $(\rho=-0.557, p=0.005)$.. Among all the kinetic parameters, IAUCtthp had the most significant correlations with SUVmax with MAD(IAUCtthp) showing the strongest correlation with SUVmax.

\section{B. Multivariate Linear Regression of SUVmax from DCE-MRI Features}

A total of 8 DCE-MRI features were initially chosen by UVA and MVA combined with LOOCV: MAD(IAUCtthp), mean(WOS), MAD(TTHP), mean(SER), SD(TTP), mean(IAUCtthp), median(IAUCtthp), and SD(WIS). These features were then used as input to the LASSO regressor to determine a regularized multivariate linear regression model for predicting SUVmax in combination with LOOCV. Out of the 8 DCE-MRI features, four features were finally selected with the LASSO: SD(WIS), SD(TTP), MAD(TTHP), and MAD(IAUCtthp). A linear combination of these 4 features constituted an in-sample multivariate linear regression model: SUVmax $\approx 16.84+3.531 \times \mathrm{SD}(\mathrm{TTP})-0.031 \times$ $\mathrm{SD}$ (WIS) $-19.37 \times \mathrm{MAD}(\mathrm{TTHP})-13.06 \times \mathrm{MAD}$ (IAUCtthp). The in-sample adjusted $\mathrm{R}^{2}$ of this model was $0.538(\mathrm{p}<0.0001)$, and its LOOCV-based adjusted $\mathrm{R}^{2}$ was $0.298(\mathrm{p}=$ 0.0006). It should be noted that the LOOCV was not only combined to select a subset of relevant features in the LASSO regression, but also to evaluate the out-of-sample performance of the LASSO regression model. The mean and SD values for the SUVmax and LASSO-selected parameters for different histopathologies and tumor stages are summarized in Table 4. No features were statistically significantly different between AC and SCC as well as between lower (I or II) and higher (III or IV) tumor stage groups.

\section{Comparison between Univariate and Multivariate Linear Regression Models}

Of the 64 DCE-MRI features, MAD(IAUCtthp) yielded the best univariate linear regression model for predicting SUVmax, which was given by: SUVmax $\approx 20.66-54.26 \times$ MAD(IAUCtthp). The in-sample adjusted $\mathrm{R}^{2}$ of this model was $0.368(\mathrm{p}=0.001)$, and its LOOCV-based adjusted $\mathrm{R}^{2}$ was $0.261(\mathrm{p}=0.006)$. Although MAD(IAUCtthp) was significant on both UVA and MVA, the LASSO-based multivariate regression model was 
better in predicting SUVmax than the best univariate linear regression model using MAD(IAUCtthp). Figure 1 shows the scatter plots of the predicted versus the measured SUVmax, comparing in-sample and out-of-sample (LOOCV) performances between the best univariate and LASSO-based multivariate regression approaches. In the out-of-sample prediction of SUVmax, one of the SUVmax (= 5.840) was predicted to be negative (= $-5.886)$ and is not displayed in Figure 1(d).

\section{Case Studies}

Figure 2(a) shows ${ }^{18}$ F-FDG-PET/CT image and LASSO-selected 4 semiquantitative kinetic parameter maps for 3 representative cases with NSCLC, where case 1 is a stage III SCC, case 2 is a stage I AC, and case 3 is a stage II SCC. SUVmax and LASSO-selected DCEMRI feature values (SD[TTP], MAD[TTHP], SD[WIS], and MAD[IAUCtthp]) of the 3 cases are shown in Figure 2(b), along with the histograms of the corresponding semiquantitative parameter maps (TTP, TTHP, WIS, and IAUCtthp). Similar histogram bin size and parameter range was used to compare data distribution among these cases. For SUVmax, case 1 showed the highest value $(=23.54)$, case 2 the second highest value (= 11.47), and case 3 the lowest value (=11.39), though the SUVmax values for cases 2 and 3 were similar. For each of the LASSO-selected DCE-MRI features, case 1 showed the highest $\mathrm{SD}$ (TTP) $(=1.434 \mathrm{~min})$ and the lowest $\mathrm{SD}$ (WIS) $\left(=3.735 \mathrm{~min}^{-1}\right)$ values, case 2 the highest $\mathrm{SD}$ (WIS) $\left(=154.3 \mathrm{~min}^{-1}\right)$ and the lowest $\mathrm{SD}(\mathrm{TTP})(=0.821 \mathrm{~min})$ values, and case 3 the highest MAD(TTHP) (0.363 min) and MAD(IAUCtthp) (0.136) values among the 3 NSCLC cases. The predicted SUVmax for the 3 NSCLC cases between the best univariate and LASSO-based multivariate linear regression models was compared. In terms of in-sample fit, SUVmax values predicted by the univariate regression model were 18.00, 19.68, and 13.28 whereas those by the LASSO regression model were 19.54, 14.06, and 11.58 for cases 1 through 3, respectively. Therefore, the SUVmax values predicted by the LASSO regression model were closer to the measured SUVmax values than those predicted by the univariate regression model.

\section{Discussion}

In this study, we investigated the correlation between tumor vascularity obtained using various semiquantitative DCE-MRI parameters and tumor metabolism obtained using SUVmax. We found that intratumoral heterogeneity in wash-in contrast kinetics was associated with tumor metabolism. This was confirmed by the fact that all the kinetic feature components (i.e., TTP, TTHP, WIS, and IAUCtthp) found in the proposed LASSO regression model were obtained during the contrast uptake phase in tumor, and the combination of their intratumoral spatial variations (i.e., SD[TTP], MAD[TTHP], SD[WIS], and MAD[IAUCtthp]) was associated with SUVmax.

LASSO-selected features contained only wash-in-phase semiquantitative parameters, i.e., TTP, TTHP, WIS, and IAUCtthp. Although semiquantitative approaches are difficult to directly relate these measures to underlying physiology because they are mixed measures, some broad correlations could exist between semiquantitative parameters and underlying physiology. For instance, the increased WIS and IAUC and the decreased TTP are likely 
related to increased vascular density and/or permeability [24]. IAUC describes how fast and how much the $\mathrm{CA}$ is delivered to the tumor and thus it reflects blood flow, blood volume, vascular permeability, and fractional interstitial volume in a mixed fashion [20, 25]. This IAUC parameter is generally preferred for analyzing DCE-MRI data because it is more stable in the presence of noise and less dependent on the temporal resolution [26]. On the other hand, TTHP is a newly derived parameter in this study to describe the half-peak time that is dependent on the curvature of the contrast wash-in. Because TTHP is the early washin time of $\mathrm{CA}$, the contrast enhancement at this time may mainly reflect blood flow and blood volume [27]. In particular, IAUCtthp played an important role in predicting SUVmax as evidenced by the fact that MAD(IAUCtthp) offered the best univariate regression model. Considering that mean(IAUCtthp) and median(IAUCtthp) were also negatively correlated with SUVmax (see Table 3), one could hypothesize that lower blood flow and blood volume may reflect a higher rate of anaerobic metabolism of glucose associated with hypoxia in NSCLC.

Even though MAD(IAUCtthp) was the best single predictor of SUVmax, using a single predictor may be insufficient to model the SUVmax precisely. Our results showed that a linear combination of the LASSO-selected predictors provides a better in-sample and out-ofsample predictive model of SUVmax than the best single predictor so that any observed change in SUVmax could result from a variety of spatiotemporal enhancement patterns of DCE-MRI in NSCLC. One criterion for evaluating the relative efficacies of univariate and multivariate regression approaches is how well their respective findings generalize beyond a particular data set to any other independently obtained data. Ideally, this type of analysis should be performed on the basis of independent training and testing data sets of large sizes. Because the small data sets were available in this study, LOOCV was a more accurate option than split-sample validation, which could overestimate prediction error for small sample sizes [28].

SUVmax is the most widely used parameter that reflects tumor glucose metabolism of the most aggressive cell component [29] and is an independent prognostic factor in NSCLC [30-34]. Uncoupling of blood flow and metabolism typically implies hypoxic stimulation of glucose metabolism and is frequently encountered in large aggressive tumors after treatment [13]. Although our results showed that SUVmax and LASSO-selected features were not significantly different between AC and SCC nor between lower (I or II) and higher (III or IV) tumor stage groups, our findings were helpful in finding the relationship between vascularity and metabolism in a pooled analysis of pretreatment NSCLC.

Only first-order statistics of the kinetic feature distributions calculated from the histogram of tumor voxel intensity values were calculated. We did not make any attempt to calculate higher order texture features. Because the statistical power of this study was limited by the small sample size, the high variability in higher order texture features could potentially make it harder to generalize variations in spatial patterns of the tumor. The tumor ROIs were drawn by a single physician. To investigate the effect of contouring variations on the model parameters, the ROIs were contracted by $1 \mathrm{~mm}$ for a subset of patient and parameter values recalculated. Our analysis suggested that contouring variations of $1 \mathrm{~mm}$ can show up to 5\% variation in median kinetic parameter values. 
There are some limitations in this study. First, the number of patients was small $(\mathrm{n}=24)$.

Second, quantitative analysis of DCE-MRI data from this study was not performed, which typically involves the convolution of an AIF with a nonlinear pharmacokinetic model of the CA concentration. We believe that the results presented here demonstrate potential feasibility of semiquantitative DCE-MRI metrics that can be efficiently incorporated with routine clinical practice because of their independence from AIF selection, prior T1mapping, and optimal fitting of the kinetic model to the tissue under investigation. Third, a pixel-by-pixel correlation analysis between DCE-MRI and ${ }^{18}$ F-FDG-PET images was not performed. The patient orientations during the two imaging (MRI and PET) examinations were different. For a pixel-by-pixel comparison, the factors that affect patient positioning would need to match each other exactly for the MRI and ${ }^{18}$ F-FDG-PET examinations. Future work will focus on a pixel-by-pixel comparison between SUVmax and DCE-MRI parameters.

\section{Conclusion}

In this study, we demonstrated the relationship between tumor vascularity and metabolism based on semiquantitative DCE-MRI parameters and ${ }^{18}$ F-FDG-PET SUVmax in NSCLC patients. Intratumoral heterogeneity in wash-in contrast kinetics was associated with tumor metabolism. A linear combination of the LASSO-selected features (SD[TTP], MAD[TTHP], SD[WIS], and MAD[IAUCtthp]) yielded better predictability of SUVmax than the best single feature (MAD[IAUCtthp]). This study will facilitate understanding of the complex relationship between tumor vascularization and metabolism, and eventually help in guiding personalized targeted therapy.

\section{Supplementary Material}

Refer to Web version on PubMed Central for supplementary material.

\section{Acknowledgments}

This study was supported by MSK Imaging and Radiation Science (IMRAS) seed grant and Cycle for Survival grants. This research was also partially supported by the NIH/NCI Cancer Center Support Grant/Core Grant (P30 CA008748). We are grateful to Mr. James Keller for his help in editing this manuscript.

\section{References}

1. Siegel R, Naishadham D, Jemal A. Cancer statistics, 2013. CA Cancer J Clin. 2013; 63(1):11-30. [PubMed: 23335087]

2. Carney DN, Hansen HH. Non-small-cell lung cancer--stalemate or progress? N Engl J Med. 2000; 343(17):1261-2. [PubMed: 11071679]

3. Oyen WJ, et al. Role of FDG-PET in the diagnosis and management of lung cancer. Expert Rev Anticancer Ther. 2004; 4(4):561-7. [PubMed: 15270660]

4. Inoue T, et al. Detecting recurrent or residual lung cancer with FDG-PET. J Nucl Med. 1995; 36(5): 788-93. [PubMed: 7738649]

5. Juweid ME, Cheson BD. Positron-emission tomography and assessment of cancer therapy. N Engl J Med. 2006; 354(5):496-507. [PubMed: 16452561]

6. De Ruysscher D, et al. Selective mediastinal node irradiation based on FDG-PET scan data in patients with non-small-cell lung cancer: a prospective clinical study. Int J Radiat Oncol Biol Phys. 2005; 62(4):988-94. [PubMed: 15989999] 
7. Yano S, et al. Molecular mechanisms of angiogenesis in non-small cell lung cancer, and therapeutics targeting related molecules. Cancer Sci. 2003; 94(6):479-85. [PubMed: 12824870]

8. Carmeliet P, Jain RK. Principles and mechanisms of vessel normalization for cancer and other angiogenic diseases. Nat Rev Drug Discov. 2011; 10(6):417-27. [PubMed: 21629292]

9. O'Connor JP, et al. Dynamic contrast-enhanced MRI in clinical trials of antivascular therapies. Nat Rev Clin Oncol. 2012; 9(3):167-77. [PubMed: 22330689]

10. Hatabu H, et al. Quantitative assessment of pulmonary perfusion with dynamic contrast-enhanced MRI. Magn Reson Med. 1999; 42(6):1033-8. [PubMed: 10571924]

11. Jackson A, Li KL, Zhu X. Semi-quantitative parameter analysis of DCE-MRI revisited: montecarlo simulation, clinical comparisons, and clinical validation of measurement errors in patients with type 2 neurofibromatosis. PLoS One. 2014; 9(3):e90300. [PubMed: 24594707]

12. Padhani AR, Miles KA. Multiparametric imaging of tumor response to therapy. Radiology. 2010; 256(2):348-64. [PubMed: 20656830]

13. Miles KA, Williams RE. Warburg revisited: imaging tumour blood flow and metabolism. Cancer Imaging. 2008; 8:81-6. [PubMed: 18390391]

14. Willinek WA, et al. Randomly segmented central k-space ordering in high-spatial-resolution contrastenhanced MR angiography of the supraaortic arteries: initial experience. Radiology. 2002; 225(2):583-8. [PubMed: 12409598]

15. Huizinga W, et al. PCA-based groupwise image registration for quantitative MRI. Med Image Anal. 2016; 29:65-78. [PubMed: 26802910]

16. Klein $\mathrm{S}$, et al. elastix: a toolbox for intensity-based medical image registration. IEEE Trans Med Imaging. 2010; 29(1):196-205. [PubMed: 19923044]

17. Lee SH, et al. Dynamic Contrast-Enhanced MRI Kinetic Parameters as Prognostic Biomarkers for Prediction of Survival of Patient with Advanced Hepatocellular Carcinoma: A Pilot Comparative Study. Acad Radiol. 2015; 22(11):1344-60. [PubMed: 26211553]

18. Chen YJ, et al. Washout gradient in dynamic contrast-enhanced MRI is associated with tumor aggressiveness of prostate cancer. J Magn Reson Imaging. 2012; 36(4):912-9. [PubMed: 22711415]

19. Lee SH, et al. Multilevel analysis of spatiotemporal association features for differentiation of tumor enhancement patterns in breast DCE-MRI. Medical Physics. 2010; 37(8):3940-3956. [PubMed: 20879557]

20. Walker-Samuel S, Leach MO, Collins DJ. Evaluation of response to treatment using DCE-MRI: the relationship between initial area under the gadolinium curve (IAUGC) and quantitative pharmacokinetic analysis. Phys Med Biol. 2006; 51(14):3593-602. [PubMed: 16825751]

21. Roberts $\mathrm{C}$, et al. Comparative study into the robustness of compartmental modeling and model-free analysis in DCE-MRI studies. J Magn Reson Imaging. 2006; 23(4):554-63. [PubMed: 16506143]

22. Degani H, Furman-Haran E. Lung cancer diagnosis using magnetic resonance imaging data obtained at three time points. Google Patents. 2012

23. Friedman J, Hastie T, Tibshirani R. Regularization Paths for Generalized Linear Models via Coordinate Descent. Journal of Statistical Software. 2010; 33(1):1-22. [PubMed: 20808728]

24. Knopp MV, et al. Pathophysiologic basis of contrast enhancement in breast tumors. J Magn Reson Imaging. 1999; 10(3):260-6. [PubMed: 10508285]

25. Evelhoch JL, et al. Magnetic resonance imaging measurements of the response of murine and human tumors to the vascular-targeting agent ZD6126. Clin Cancer Res. 2004; 10(11):3650-7. [PubMed: 15173071]

26. Jesberger JA, et al. Model-free parameters from dynamic contrast-enhanced-MRI: sensitivity to EES volume fraction and bolus timing. J Magn Reson Imaging. 2006; 24(3):586-94. [PubMed: 16892197]

27. Cuenod CA, Balvay D. Perfusion and vascular permeability: basic concepts and measurement in DCE-CT and DCE-MRI. Diagn Interv Imaging. 2013; 94(12):1187-204. [PubMed: 24211260]

28. Molinaro AM, Simon R, Pfeiffer RM. Prediction error estimation: a comparison of resampling methods. Bioinformatics. 2005; 21(15):3301-7. [PubMed: 15905277] 
29. Berghmans T, et al. Primary tumor standardized uptake value (SUVmax) measured on fluorodeoxyglucose positron emission tomography (FDG-PET) is of prognostic value for survival in non-small cell lung cancer (NSCLC): a systematic review and meta-analysis (MA) by the European Lung Cancer Working Party for the IASLC Lung Cancer Staging Project. J Thorac Oncol. 2008; 3(1):6-12. [PubMed: 18166834]

30. Horne ZD, et al. Pretreatment SUVmax predicts progression-free survival in early-stage non-small cell lung cancer treated with stereotactic body radiation therapy. Radiat Oncol. 2014; 9:41. [PubMed: 24479954]

31. Imamura Y, et al. Prognostic value of SUVmax measurements obtained by FDG-PET in patients with non-small cell lung cancer receiving chemotherapy. Lung Cancer. 2011; 71(1):49-54. [PubMed: 20430470]

32. Shirai K, et al. Maximum standardized uptake value on FDG-PET predicts survival in stage I nonsmall cell lung cancer following carbon ion radiotherapy. Oncol Lett. 2017; 13(6):4420-4426. [PubMed: 28588712]

33. Nappi A, et al. [F-18] FDG-PET/CT parameters as predictors of outcome in inoperable NSCLC patients. Radiol Oncol. 2015; 49(4):320-6. [PubMed: 26834517]

34. Yoo Ie R, et al. Prognostic value of SUVmax and metabolic tumor volume on 18F-FDG PET/CT in early stage non-small cell lung cancer patients without LN metastasis. Biomed Mater Eng. 2014; 24(6):3091-103. [PubMed: 25227018] 


\section{Summary}

Balance between vascularity and glucose metabolism in tumor could prove to be an important indicator of its biological status and resistance to treatment. This study evaluates the use of semiquantitative dynamic contrast-enhanced MRI parameters for predicting the ${ }^{18}$ F-FDG-PET maximum standardized uptake value in non-small cell lung cancer (NSCLC). It was found that intratumoral heterogeneity in wash-in contrast kinetics is associated with tumor metabolism. Investigating vascular-metabolic relationship will help in guiding personalized targeted therapy in NSCLC. 


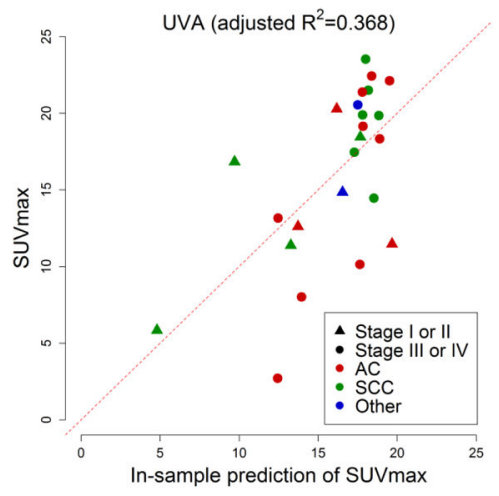

(a)

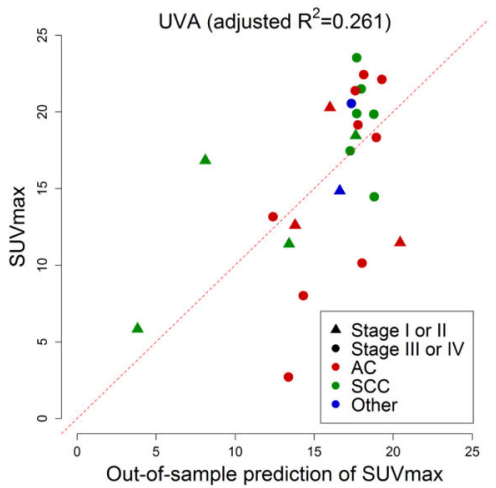

(c)

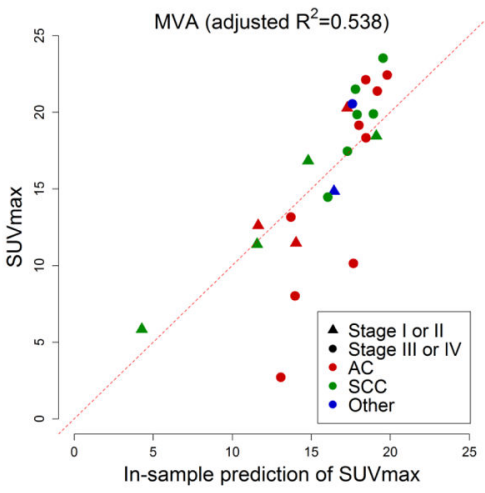

(b)

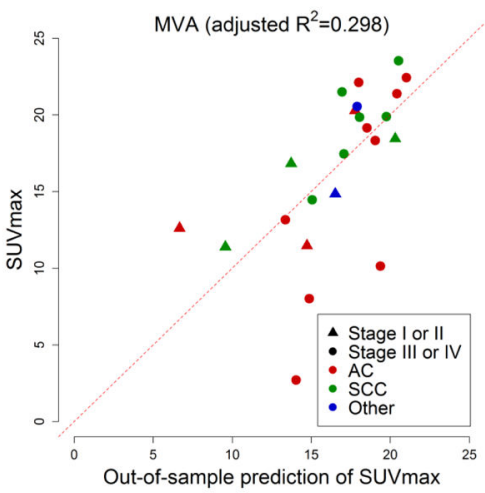

(d)

Figure 1.

Scatter plots of the predicted (horizontal axis) versus measured SUVmax (vertical axis), comparing in-sample (a and b) and out-of-sample (LOOCV) goodness-of-fit (c and d) between the best univariate (a and c) and LASSO-based multivariate regression approaches ( $b$ and d). The MVA using the LASSO regression was better (i.e., higher adjusted $\mathrm{R}^{2}$ ) than the UVA using simple univariate linear regression. 


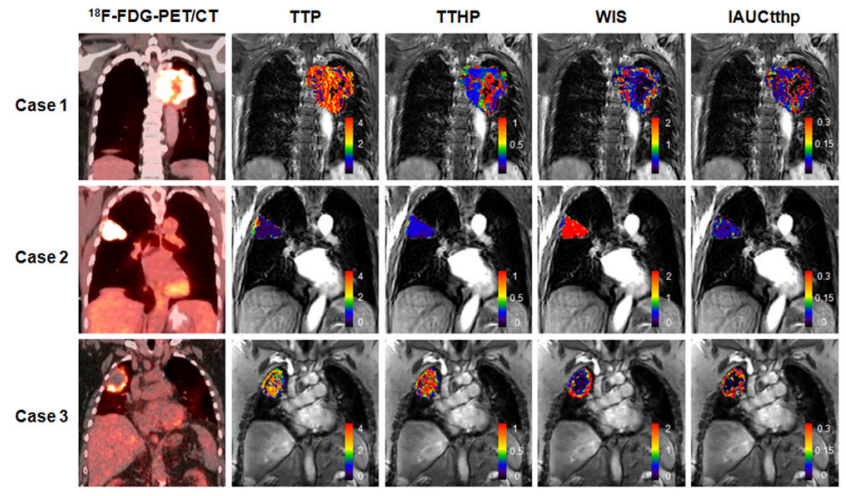

(a)
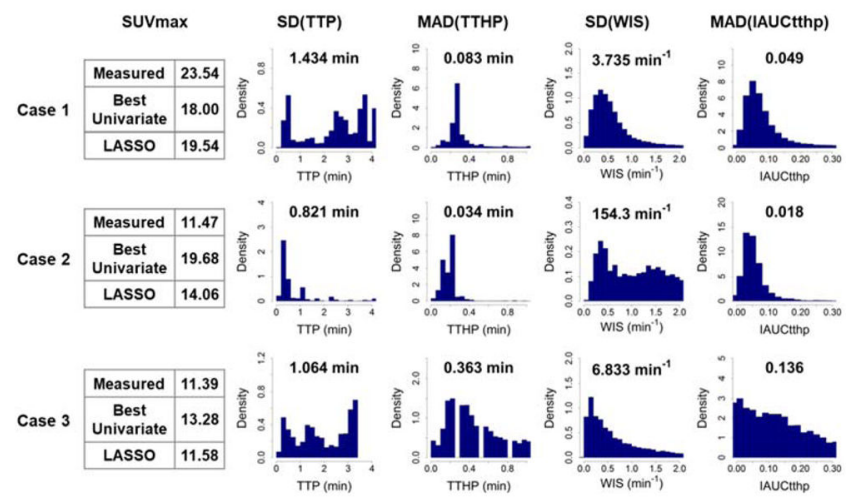

(b)

Figure 2.

Three representative cases with NSCLC showing (a) ${ }^{18}$ F-FDG-PET/CT image and LASSOselected 4 semiquantitative DCE-MRI parameter maps (time-to-peak [TTP], time-to-halfpeak [TTHP], wash-in-slope [WIS], and initial area under the curve until TTHP [IAUCtthp]) as well as (b) measured and predicted SUVmax and spatiotemporal DCE-MRI features (SD[TTP], MAD[TTHP], SD[WIS], and MAD[IAUCtthp]), along with histograms of their corresponding semiquantitative kinetic parameters (TTP, TTHP, WIS, and IAUCtthp). 


\section{Table 1}

Patient characteristics

\begin{tabular}{|c|c|c|}
\hline \multicolumn{2}{|l|}{ Parameter } & \multirow{2}{*}{$\frac{\text { No. of Patients }}{14}$} \\
\hline \multirow{2}{*}{ Sex } & M & \\
\hline & $\mathrm{F}$ & 10 \\
\hline \multirow{2}{*}{ Age } & Mean & $65.25 \mathrm{y}$ \\
\hline & Range & $40-89 y$ \\
\hline \multirow{2}{*}{ Weight } & Mean & $77.33 \mathrm{~kg}$ \\
\hline & Range & $47-113 \mathrm{~kg}$ \\
\hline \multirow{3}{*}{ RT (Definitive or sequential CRT) } & Definitive RT only & 12 \\
\hline & Sequential CRT & 10 \\
\hline & N/A & 2 \\
\hline \multirow{2}{*}{ Tumor Volume (CC) } & Mean & $154.2 \mathrm{cc}$ \\
\hline & Range & $4.5-1130 \mathrm{cc}$ \\
\hline \multirow{2}{*}{ RT technique } & Standard fraction & 12 \\
\hline & SBRT & 12 \\
\hline \multirow{2}{*}{ Tumor stage } & I or II & 8 \\
\hline & III or IV & 16 \\
\hline \multirow{4}{*}{ Histopathology } & $\mathrm{AC}$ & 12 \\
\hline & SCC & 10 \\
\hline & ASCC & 1 \\
\hline & NOS & 1 \\
\hline
\end{tabular}

$\mathrm{M}=$ male $; \mathrm{F}=$ female $; \mathrm{AC}=$ adenocarcinoma $\mathrm{SCC}=$ squamous cell carcinoma $; \mathrm{ASCC}=$ adenosquamous cell carcinoma NOS = not otherwise specified; $\mathrm{RT}=$ radiation therapy $\mathrm{CRT}=$ Chemoradiation $; \mathrm{SBRT}=$ stereotactic body radiation therapy 


\section{Table 2}

\section{Semiquantitative metrics of DCE-MRI}

\begin{tabular}{|c|c|c|}
\hline Parameter & Definition & Unit \\
\hline Peak enhancement (PE) & $\begin{array}{l}\text { Peak relative signal enhancement (RSE), i.e., maximum signal difference (MSD) divided by } \\
\text { signal baseline (SB), where MSD is the difference between the signal intensity at its maximum } \\
\text { and SB }\end{array}$ & \\
\hline Time-to-peak (TTP) & Time at which PE occurs, minus bolus arrival time (BAT) in the feeding artery & $\min$ \\
\hline Time-to-half-peak (TTHP) & Time at which half PE occurs before time at which PE occurs, mus BAT in the feeding artery & $\min$ \\
\hline Wash-in-slope (WIS) & PE divided by TTP & $\min ^{-1}$ \\
\hline Wash-out-slope (WOS) & $\begin{array}{l}\text { Difference between PE and RSE at the last time point }\left(T_{\text {end }}\right) \text {, which is divided by difference } \\
\text { between } T_{\text {end }} \text { and time at which PE occurs, if the PE does not occur at } T_{\text {end }} \text {; Otherwise zero }\end{array}$ & $\min ^{-1}$ \\
\hline Initial gradient (IG) & Gradient calculated by linear regression of all the RSEs between $10 \% \mathrm{PE}$ and $70 \% \mathrm{PE}$ & $\min ^{-1}$ \\
\hline Washout gradient (WG) & $\begin{array}{l}\text { Gradient calculated by linear regression of all the RSEs taken between } 1 \text { and } 2 \text { min elapsed from } \\
\text { BAT in the feeding artery }\end{array}$ & $\min ^{-1}$ \\
\hline Signal enhancement ratio (SER) & RSE at 0.5 min divided by RSE at $2.5 \mathrm{~min}$, elapsed from BAT in the feeding artery & \\
\hline Initial area under the curve (IAUC) & Area under the RSE curve until a stipulated time point, elapsed from BAT in the feeding artery & \\
\hline
\end{tabular}

Int J Radiat Oncol Biol Phys. Author manuscript; available in PMC 2019 November 15. 
Table 3

Spearman rank correlation coefficients between DCE-MRI features and ${ }^{18}$ F-FDG-PET SUVmax

\begin{tabular}{ccccc}
\hline & \multicolumn{4}{c}{ Spatial feature } \\
\cline { 2 - 5 } Kinetic feature & Mean & Median & SD & MAD \\
\hline PE & -0.268 & -0.137 & -0.176 & -0.347 \\
TTP & 0.152 & 0.139 & 0.230 & 0.184 \\
TTHP & -0.277 & -0.351 & -0.152 & -0.367 \\
WIS & -0.291 & -0.232 & -0.284 & -0.230 \\
WOS & $-\mathbf{0 . 4 5 6}$ & -0.171 & -0.323 & -0.207 \\
IG & 0.185 & 0.318 & -0.067 & 0.177 \\
WG & -0.009 & 0.158 & -0.179 & -0.370 \\
SER & -0.381 & -0.053 & 0.037 & -0.286 \\
IAUC30 & -0.160 & 0.138 & -0.137 & -0.079 \\
IAUC60 & -0.095 & 0.148 & -0.156 & -0.071 \\
IAUC90 & -0.106 & 0.127 & -0.160 & -0.190 \\
IAUC120 & -0.111 & 0.120 & -0.159 & -0.243 \\
IAUC150 & -0.110 & 0.128 & -0.153 & -0.264 \\
IAUC180 & -0.056 & 0.182 & -0.140 & -0.311 \\
IAUCttp & 0.055 & 0.134 & 0.096 & 0.114 \\
IAUCtthp & $-\mathbf{0 . 4 3 9 *}$ & $\mathbf{- 0 . 5 4 3}{ }^{\dagger}$ & -0.232 & $\mathbf{- 0 . 5 5 7}{ }^{\dagger}$ \\
\hline
\end{tabular}

$\mathrm{PE}=$ peak enhancement; TTP = time-to-peak; TTHP = time-to-half-peak; WIS = wash-in-slope $;$ WOS = wash-out-slope; IG = initial gradient; WG = washout gradient; SER = signal enhancement ratio; IAUC30, IAUC60, IAUC90, IAUC120, IAUC150, IAUC180, IAUCttp and IAUCtthp = initial area under the curve taken up to 30, 60, 90, 120,150, 180 seconds, TTP and TTHP, respectively; SD = standard deviation; MAD = median absolute deviation.

p $<0.05$

$t_{\mathrm{p}}<0.01$. 


\section{Table 4}

Mean \pm SD of the SUVmax and LASSO-selected DCE-MRI features in MVA grouped in terms of histopathology and tumor stage

\begin{tabular}{ccccc}
\hline & \multicolumn{2}{c}{ Histopathology } & \multicolumn{2}{c}{ Tumor stage } \\
\cline { 2 - 5 } Feature & AC $(\mathbf{n = 1 2})$ & SCC $(\mathbf{n = 1 0})$ & I or II (n=8) & III or IV (n=16) \\
\hline SUVmax & $15.15 \pm 6.380$ & $16.92 \pm 5.210$ & $13.97 \pm 4.624$ & $17.17 \pm 5.924$ \\
SD(TTP) (min) & $1.149 \pm 0.295$ & $1.112 \pm 0.304$ & $1.111 \pm 0.313$ & $1.150 \pm 0.273$ \\
MAD(TTHP) (min) & $0.130 \pm 0.096$ & $0.176 \pm 0.190$ & $0.207 \pm 0.206$ & $0.123 \pm 0.083$ \\
SD(WIS) (min $\left.{ }^{-1}\right)$ & $36.03 \pm 53.52$ & $12.15 \pm 15.04$ & $47.78 \pm 62.97$ & $11.46 \pm 13.04$ \\
MAD(IAUCtthp) & $0.076 \pm 0.050$ & $0.097 \pm 0.087$ & $0.124 \pm 0.088$ & $0.064 \pm 0.041$ \\
\hline
\end{tabular}

TTP = time-to-peak; TTHP = time-to-half-peak; WIS = wash-in-slope; IAUCtthp = initial area under the curve taken up to TTHP; SD = standard deviation; $\mathrm{MAD}=$ median absolute deviation; $\mathrm{AC}=$ adenocarcinoma; $\mathrm{SCC}=$ squamous cell carcinoma 